\title{
Ceramide bei Depression
}

In den letzten Jahren haben wir in enger Kooperation mit der Arbeitsgruppe von Erich Gulbins, Essen, begonnen, die Rollen der sauren Sphingomyelinase (acid sphingomyelinase, ASM) und des Ceramidsystems bei schwerer Depression zu analysieren. Die ASM katalysiert den Abbau von Sphingomyelin zu Phosphorylcholin und Ceramid bei saurem pH-Wert. Das Enzym ist in Lysosomen lokalisiert, aber auch auf kleinen sauren Domänen der äußeren Schicht der Plasmamembran präsent. Die letztgenannte Form zeigt wichtige Signalisierungsfunktionen. Abhängig von ihrer Glycosylierung wird die ASM auch in den extrazellulären Raum sezerniert.

Die Aktivität der ASM an der Zelloberfläche führt zur Bildung von Ceramid in der äußeren Schicht der Zellmembran. Ceramid ist besonders lipophil; Ceramidmoleküle bilden spontan kleine Ceramid-angereicherte Membrandomänen, die zu großen mit Ceramid angereicherten Membranplattformen verschmelzen. Diese Plattformen fangen Rezeptormoleküle und initiieren Stresssignale. Obwohl die Regulation der ASM nicht gut charakterisiert ist, deutet vieles darauf hin, dass das Enzym durch Redoxmechanismen, insbesondere über ein redoxempfindliches Cystein reguliert wird. Einige Wachstumsfaktoren, z. B. VEGF, hemmen die ASM.

Verschiedene Beobachtungen legen eine bedeutende Rolle des ASM/Ceramid-Systems bei der Depression nahe: Viele trizyklische und tetrazyklische Antidepressiva wie Desipramin, Imipramin oder Amitriptylin hemmen die Aktivität der ASM funktionell [1,2]. Bislang wurde diese Wirkung von Antidepressiva nicht mit den therapeutischen Efekten in Verbindung gebracht. Wir konnten jedoch zeigen, dass therapeutische Konzentrationen der Antidepressiva Amitriptylin und Fluoxetin die
ASM-Aktivität und Ceramid-Konzentration im Hippocampus reduzieren. Diese Aktion vermittelt die therapeutischen Wirkungen von Antidepressiva im Tiermodell, insbesondere erhöhte neuronale Proliferation, Reifung und Überleben sowie normalisiertes Verhalten im Mausmodell der stressinduzierten Depression. Diese Studien verwendeten genetisch veränderte Tiere, denen entweder die ASM fehlten oder die sie überexprimierten. Aber auch eine pharmakologisch-induzierte Erhöhung der Ceramid-Konzentration im Hippokampus, entweder über Mikroinjektion von C16Ceramid (einem natürlichen Ceramid) oder durch PDMP, führte zu einem depressionsähnlichen Verhalten bei Mäusen, auch in Abwesenheit von Stress. Dies deutet darauf hin, dass erhöhte Ceramidspiegel auch ohne Stress Symptome einer Depression auslösen können und dass Antidepressiva zumindest teilweise über eine Reduktion der Ceramidspiegel im Hippocampus wirken. Chronisch unvorhersehbarer Stress führte zu erhöhten Hippokampus-Ceramid-Spiegeln [3]. Offensichtlich reguliert das aus Endothelzellen freigesetzte Ceramid neuronale Stammzellen im Hippokampus [4].

Es ist derzeit unbekannt, ob Patienten mit schwerer Depression erhöhte Ceramidspiegel im Hippokampus aufweisen. In Blutproben wurden erhöhte Aktivitäten der ASM und Ceramid-Konzentrationen bei schwerer depressiver Störung [5], depressiven Syndromen und bei posttraumatischer Belastungsstörung gefunden. Endogene Veränderungen im Ceramidstoffwechsel scheinen daher auch beim Menschen mit Depression assoziiert zu sein.

Künftige Studien müssen klären, wie Ceramid eine Depression vermittelt und ob die
Menge an Ceramid den Schweregrad einer Depression bestimmt.

Interessenkonflikte

Keine

\section{Autor}

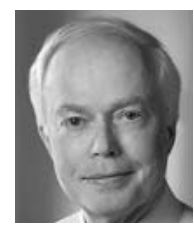

Johannes Kornhuber

Psychiatrische und Psychotherapeutische Klinik, Erlangen

Korrespondenzadresse

Prof. Dr. Johannes Kornhuber

Direktor

Psychiatrische und

Psychotherapeutische Klinik

Schwabachanlage 6

91054 Erlangen

Johannes.Kornhuber@uk-erlangen.de

\section{Literatur}

[1] Kornhuber J, Tripal P, Reichel M et al. Identification of new functional inhibitors of acid sphingomyelinase using a structure-property-activity relation model. J Med Chem 2008; 51: 219-237

[2] Kornhuber J, Muehlbacher M, Trapp S et al. Identification of novel functional inhibitors of acid sphingomyelinase. PLOS ONE 2011; 6: e23852

[3] Gulbins E, Palmada M, Reichel $M$ et al. Acid sphingomyelinase/ceramide system mediates effects of antidepressant drugs. Nat Med 2013; 19: 934-938

[4] Gulbins A, Grassmé H, Hoehn R et al. Regulation of neuronal stem cell proliferation in the hippocampus by endothelial ceramide. Cell Physiol Biochem 2016; 39: 790-801

[5] Kornhuber J, Medlin A, Bleich S et al. High activity of acid sphingomyelinase in major depression. J Neural Transm 2005; 112: 15831590 